\title{
Noninvasive evaluation of left ventricular force-frequency relationships by measuring carotid arterial wave intensity during exercise stress
}

\author{
Midori Tanaka - Motoaki Sugawara • \\ Yasuo Ogasawara · Isao Suminoe • Tadafumi Izumi • \\ Kiyomi Niki • Fumihiko Kajiya
}

Received: 19 February 2014/Accepted: 14 May 2014/Published online: 24 June 2014

(C) The Japan Society of Ultrasonics in Medicine 2014

\begin{abstract}
Background and purpose Estimation of the contractility of the left ventricle during exercise is important in drawing up a protocol of cardiac rehabilitation. It has been demonstrated that color Doppler- and echo tracking-derived carotid arterial wave intensity is a sensitive index of global left ventricular (LV) contractility. We assessed the feasibility of measuring carotid arterial wave intensity and determining force-frequency (contractility-heart rate) relations (FFRs) during exercise totally noninvasively.

Methods We measured carotid arterial wave intensity with a combined color Doppler and echo tracking system in 25 healthy young male volunteers (age $20.8 \pm 1.2$ years) at rest and during exercise. FFRs were constructed by plotting the maximum value of wave intensity $\left(\mathrm{WD}_{1}\right)$ against heart rate (HR).
\end{abstract}

M. Tanaka $(\bowtie) \cdot$ M. Sugawara

Faculty of Health Care Sciences, Himeji Dokkyo University, 7-2-1 Kamiohno, Himeji, Hyogo 670-8524, Japan

e-mail: mirakulu17.nikkori17@gmail.com

Y. Ogasawara · F. Kajiya

Department of Medical Engineering, Kawasaki University of Medical Welfare, Kurashiki, Japan

I. Suminoe

Clinical Laboratory, Japanese Red Cross Society, Himeji

Hospital, Himeji, Japan

T. Izumi

Department of Physical Therapy, Health Sciences University of Hokkaido, Ishikari, Japan

K. Niki

Biomedical Engineering Department, Tokyo City University,

Tokyo, Japan
Results We first confirmed that HR increased linearly with an increase in work load in each subject $\left(r^{2}=0.95 \pm 0.04\right) . \mathrm{WD}_{1}$ increased linearly with an increase in HR. The goodness-of-fit of the regression line of $\mathrm{WD}_{1}$ on $\mathrm{HR}$ in each subject was very high $\left(r^{2}=0.48-0.94, p<0.0001\right.$, respectively). The slope of the $\mathrm{WD}_{1}$-HR relation ranged $0.30-2.20\left[\mathrm{~m} / \mathrm{s}^{3}\right.$ (beat $\left.\left./ \mathrm{min}\right)\right]$. Conclusions Global LV FFRs can be generated in healthy young volunteers with an entirely noninvasive combination of exercise and wave intensity. These data should show the potential usefulness of the FFR in the context of cardiac rehabilitation.

Keywords Force-frequency relation .

Echocardiography $\cdot$ Wave intensity $\cdot$ Exercise

\section{Introduction}

Evaluation of the changes in the contractile state of the left ventricle during exercise is an important, and as yet not easy, goal in the procedure for cardiac rehabilitation. The positive inotropic effect of increasing the frequency of contraction in isolated cardiac muscles has been known as the Bowditch effect or the staircase phenomenon. The positive inotropic effect of increased heart rate (HR) produced by pacing also has been demonstrated in healthy human subjects, which has been called the force-frequency relation (FFR) [1, 2]. In conventional methods of obtaining FFRs, the maximum rate of left ventricular (LV) pressure rise (peak $\mathrm{d} P / \mathrm{d} t$ ) measured with a catheter-tipped micromanometer was used as an index of cardiac contractility, and atrial pacing was used to change $\mathrm{HR}$ (peak $\mathrm{d} P / \mathrm{d} t-\mathrm{HR}$ relation). It has also been reported that the FFR is significantly affected in hearts with a variety of diseases [3-7]. 


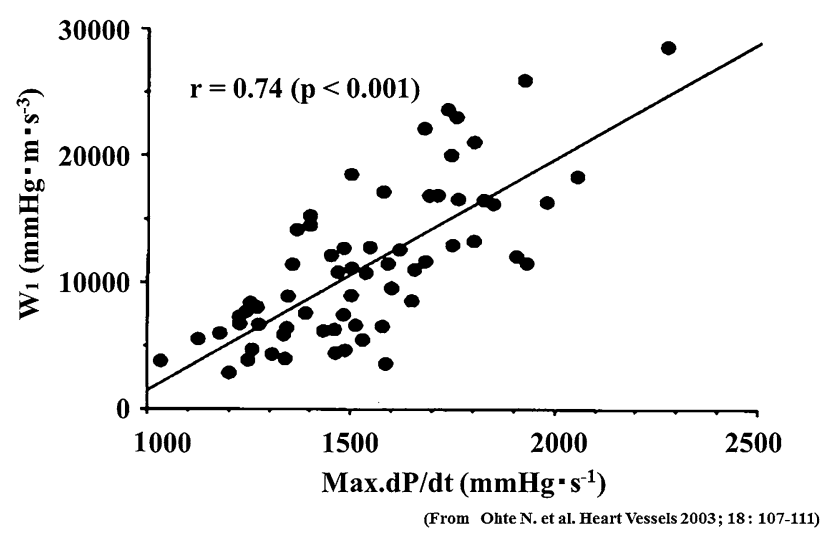

Fig. 1 Relation between $\mathrm{W}_{1}$ obtained by carotid arterial echography and $\max \mathrm{d} P / \mathrm{d} t$ obtained with a catheter-tipped micromanometer (From Ohte $\mathrm{N}$ et al. [9])

During exercise, HR increases with an increase in workload; therefore, atrial pacing is not needed for changing HR. It has also been demonstrated that the peak $\mathrm{d} P / \mathrm{d} t-\mathrm{HR}$ relation is markedly enhanced (the slope is increased) during exercise compared with during pacing in normal hearts, but the enhancement is limited in diseased hearts [8]. The FFR obtained by exercise may have higher power to discriminate cardiac contractile states than that obtained by pacing. In spite of its potential for evaluating cardiac function during exercise, peak $\mathrm{d} P / \mathrm{d} t-\mathrm{HR}$ relation has not been widely used because of its invasive nature. We have reported that carotid arterial wave intensity (WI) noninvasively measured with a combined system of color Doppler and echo tracking is a sensitive index of global LV contractile function (Fig. 1) [9, 10].

In this study, we assessed the usefulness of WI to measure LV contractility and FFRs during exercise in healthy subjects, and evaluated the feasibility of an entirely noninvasive method for demonstration of the global LV FFR.

\section{Materials and methods}

Definition of wave intensity in terms of diameterchange

Wave intensity (WI) is a hemodynamic index, which is defined as

$\mathrm{WI}=(\mathrm{d} P / \mathrm{d} t)(\mathrm{d} U / \mathrm{d} t)$

where $\mathrm{d} P / \mathrm{d} t$ and $\mathrm{d} U / \mathrm{d} t$ are the derivatives of blood pressure $(P)$ and velocity $(U)$ with respect to time, respectively. The maximum value of WI during a cardiac cycle $\left(\mathrm{W}_{1}\right)$ significantly correlates with peak $\mathrm{d} P / \mathrm{d} t$ (or $\max \mathrm{d} P / \mathrm{d} t$ ) (Fig. 1) [9].

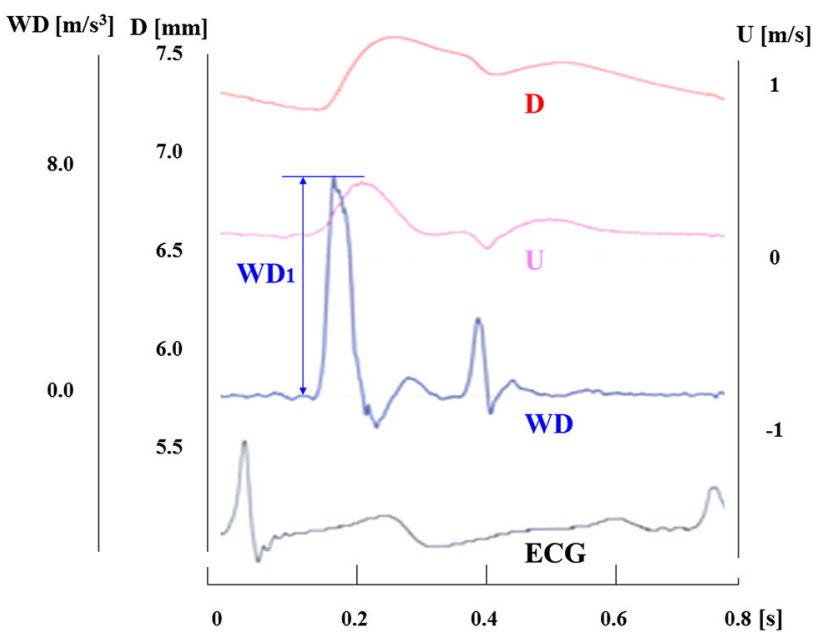

Fig. 2 Representative recordings of carotid arterial diameter $(D)$ and blood flow velocity $(U)$, and calculated wave intensity (WD) in a healthy human. WD is the wave intensity defined by using $D$ as $\mathrm{WD}=(1 / D)(\mathrm{d} D / \mathrm{d} t)(\mathrm{d} U / \mathrm{d} t)$

In our method of obtaining carotid arterial WI, the carotid diameter-change waveform was used as a surrogate for carotid pressure waveform. Using the diameter-change waveform directly, we can also define another wave intensity (WD) as

$\mathrm{WD}=(1 / D)(\mathrm{d} D / \mathrm{d} t)(\mathrm{d} U / \mathrm{d} t)$

where $D$ is the diameter. We can obtain the relation of WD to WI as follows:

The definition of the stiffness parameter, $\beta$, is written as $\beta=\left(\ln P-\ln P_{\mathrm{d}}\right) /\left[\left(D-D_{\mathrm{d}}\right) / D_{\mathrm{d}}\right]$,

where $D$ is the diameter at pressure $P$, and $D_{\mathrm{d}}$ is the diameter at the end-diastolic pressure $P_{\mathrm{d}}$. The above equation gives:

$\beta\left(D-D_{\mathrm{d}}\right) / D_{\mathrm{d}}=\ln P-\ln P_{\mathrm{d}}$.

The differentials of both sides of the above equation give $\beta \mathrm{d} D / D_{\mathrm{d}}=\mathrm{d} P / P$.

Since the difference between $D$ and $D_{\mathrm{d}}$ is small (Fig. 2), $D_{\mathrm{d}}$ can be replaced by $D$. Then, we obtain

$\mathrm{d} D / D=\mathrm{d} P / \beta P$,

which gives

$(1 / D)(\mathrm{d} D / \mathrm{d} t)=(1 / \beta P)(\mathrm{d} P / \mathrm{d} t)$,

where $\mathrm{d} D / \mathrm{d} t$ and $\mathrm{d} P / \mathrm{d} t$ are the time derivatives of $D$ and $P$, respectively. Substituting Eq. 3 into Eq. 2 and using Eq. 1, we obtain

$\mathrm{WD}=(1 / \beta P) \mathrm{WI}$

Figure 2 shows the WD waveform calculated from the diameter-change waveform and the velocity waveform. 


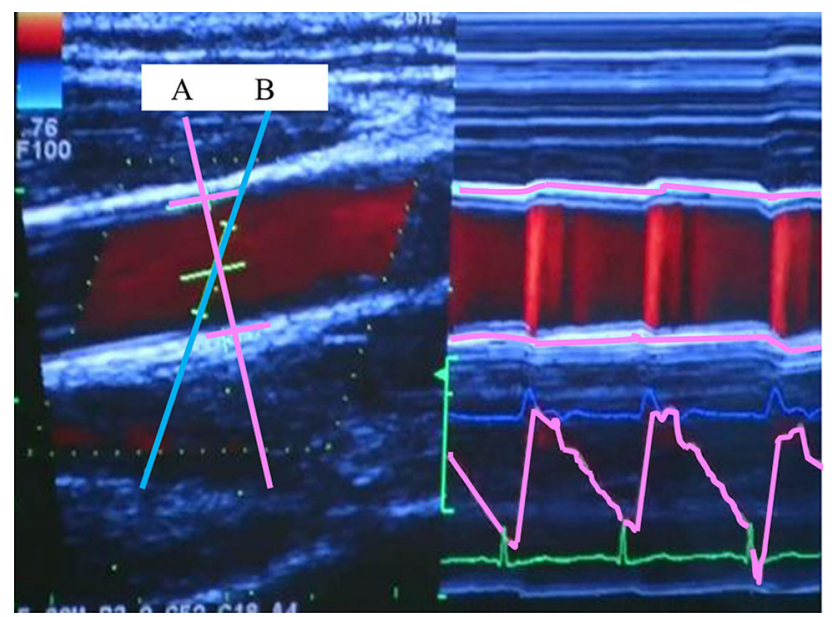

Fig. 3 Measurements of diameter-change waveform and blood velocity. Left Long axis view of the common carotid artery and ultrasound beams. By setting the tracking positions displayed as small pink bars on the echo tracking beam (line $A$ ) to arterial walls, echo tracking automatically starts. The blood flow velocity averaged along the Doppler beam (line B) crossing the artery was measured using range-gated color Doppler signals. Right The diameter-change waveform, which is calculated by subtracting the distance to the near wall from that to the far wall, is displayed on the M-mode view. The blood flow velocity waveform is also displayed on the M-mode view

According to Eq. 4, the maximum value of carotid arterial WD during a cardiac cycle $\left(\mathrm{WD}_{1}\right)$ correlates with the maximum value of $\mathrm{WI}\left(\mathrm{W}_{1}\right)$. Therefore, $\mathrm{WD}_{1}$ correlates with peak $\mathrm{d} P / \mathrm{d} t$ as $\mathrm{W}_{1}$ does (Fig. 1). WD is obtained by measuring $U$ and $D$ without measuring upper arm pressure (Fig. 3), which is easier to perform during exercise. The details of the method of measurements were described elsewhere [11].

Thus, we can regard the $\mathrm{WD}_{1}$-HR relation as the FFR.

\section{Subjects}

We studied 25 healthy male volunteers (mean age $20.8 \pm 1.2$ years, age range $19-23$ years) (Table 1$)$. We obtained informed consent from all the subjects. Approval from the Ethics Committee of Himeji Dokkyo University was obtained before study initiation.

\section{Protocol}

Subjects were asked to refrain from caffeine, alcohol, and exercise during the 24-h period before the test. Before the measurements of $\mathrm{WD}_{1}$ and $\mathrm{HR}$, the subjects were laid down the supine position for $10 \mathrm{~min}$. The location to be measured was the right common carotid artery at about $2 \mathrm{~cm}$ proximal to the carotid bulb. We used scanning in the long axis view, and obtained a B-mode image of a longitudinal section of the artery (Fig. 3, left). With the B- and
Table 1 Baseline data of all the subjects

\begin{tabular}{llllllc}
\hline Subject & $\begin{array}{l}\text { Age } \\
\text { (years })\end{array}$ & $\begin{array}{l}\text { Height } \\
(\mathrm{cm})\end{array}$ & $\begin{array}{l}\text { Weight } \\
(\mathrm{kg})\end{array}$ & $\begin{array}{l}\mathrm{BMI} \\
\left(\mathrm{kg} / \mathrm{m}^{2}\right)\end{array}$ & $\begin{array}{l}\text { HR at } \\
\text { rest } \\
(\mathrm{bpm})\end{array}$ & $\begin{array}{l}\mathrm{WD}_{1} \text { at } \\
\text { rest } \\
\left(\mathrm{m} / \mathrm{s}^{3}\right)\end{array}$ \\
\hline $\mathrm{a}$ & 23 & 171 & 64 & 21.9 & 65 & 27.77 \\
$\mathrm{~b}$ & 23 & 171 & 64 & 21.9 & 74 & 32.19 \\
$\mathrm{c}$ & 21 & 175 & 62 & 20.2 & 61 & 10.17 \\
$\mathrm{~d}$ & 21 & 163 & 55 & 20.7 & 84 & 22.94 \\
$\mathrm{e}$ & 21 & 170 & 60 & 20.8 & 74 & 19.00 \\
$\mathrm{f}$ & 20 & 172 & 65 & 22.0 & 65 & 20.71 \\
$\mathrm{~g}$ & 21 & 173 & 54 & 18.0 & 82 & 8.95 \\
$\mathrm{~h}$ & 19 & 175 & 63 & 20.6 & 78 & 18.00 \\
$\mathrm{i}$ & 21 & 171 & 87 & 29.8 & 73 & 22.40 \\
$\mathrm{j}$ & 22 & 172 & 66 & 22.3 & 74 & 23.00 \\
$\mathrm{k}$ & 19 & 177 & 71 & 22.7 & 61 & 9.10 \\
$\mathrm{l}$ & 19 & 160 & 56 & 21.9 & 88 & 50.00 \\
$\mathrm{~m}$ & 19 & 180 & 69 & 21.3 & 62 & 19.00 \\
$\mathrm{n}$ & 21 & 173 & 70 & 23.4 & 62 & 22.40 \\
$\mathrm{o}$ & 22 & 170 & 60 & 20.8 & 79 & 17.40 \\
$\mathrm{p}$ & 21 & 168 & 63 & 22.3 & 76 & 17.10 \\
$\mathrm{q}$ & 21 & 159 & 55 & 21.8 & 104 & 23.60 \\
$\mathrm{r}$ & 22 & 168 & 85 & 30.1 & 74 & 25.50 \\
$\mathrm{~s}$ & 21 & 173 & 53 & 17.7 & 74 & 36.50 \\
$\mathrm{t}$ & 19 & 160 & 52 & 20.3 & 66 & 33.74 \\
$\mathrm{u}$ & 22 & 176 & 90 & 29.1 & 84 & 13.66 \\
$\mathrm{v}$ & 22 & 174 & 78 & 25.8 & 71 & 34.98 \\
$\mathrm{w}$ & 19 & 177 & 60 & 19.2 & 71 & 44.51 \\
$\mathrm{x}$ & 21 & 165 & 50 & 18.4 & 73 & 42.02 \\
$\mathrm{y}$ & 21 & 172 & 54 & 18.3 & 86 & 27.29 \\
Mean & 20.8 & 170.6 & 64.2 & 22.0 & 74.3 & 24.9 \\
$\mathrm{SD}$ & 1.2 & 5.4 & 10.8 & 3.3 & 9.9 & 10.6 \\
\hline & & & & & &
\end{tabular}

M-mode scans displayed simultaneously on a split screen, the echo tracking system tracked the vessel wall movements to produce displacement waveforms of the anterior and posterior artery walls (Fig. 3, right). This gave the diameter-change waveforms.

After the measurements of $\mathrm{WD}_{1}$ and $\mathrm{HR}$ at rest, gradual bicycle exercise was performed starting at an initial workload of $20 \mathrm{~W}$ and lasting for $2 \mathrm{~min}$; thereafter, the workload was increased stepwise by $20 \mathrm{~W}$ at $1 \mathrm{~min}$ intervals. Electrocardiogram was continuously monitored. The criteria for the endpoint included increase of heart rate to $[(220$-age $) \times 0.8(\mathrm{bpm})]$, and achievement of maximum fatigue or the impossibility of continuing exercise. We measured $\mathrm{WD}_{1}$ and $\mathrm{HR}$ during the exercise.

Statistical analysis

The obtained data are expressed as mean \pm standard deviation. The scatter diagram of the points $\left(\mathrm{WD}_{1}, \mathrm{HR}\right)$ for 
the data during exercise from each subject was analyzed by the linear regression method, and the regression line was regarded as the FFR. A value of $p<0.05$ was considered statistically significant.

\section{Results}

The baseline data from the subjects are shown in Table 1 . We first confirmed that HR increased linearly with an increase in work load in all the subjects $\left(r^{2}=0.95 \pm 0.04\right)$ (Fig. 4). $\mathrm{WD}_{1}$ increased linearly with an increase in $\mathrm{HR}$

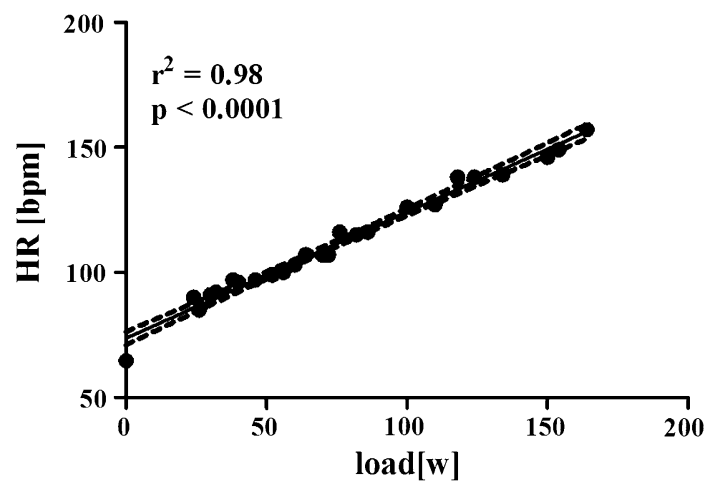

Fig. 4 A representative relation between heart rate (HR) (bpm) and work load $(W)$ in healthy male subjects. The solid line indicates the linear regression line, and the dotted curves show the $95 \%$ confidence band of the regression line
(Fig. 5). The goodness-of-fit of the regression line of $\mathrm{WD}_{1}$ on HR in each subject was very high $\left(r^{2}=0.8 \pm 0.1\right.$, $p<0.0001$, respectively). The slope of the $\mathrm{WD}_{1}-\mathrm{HR}$ relation ranged from 0.30 to $2.20\left[\mathrm{~m} / \mathrm{s}^{3}\right.$ (beat $/ \mathrm{min}$ )] $\left(\right.$ mean \pm SD: $1.0 \pm 0.4\left[\mathrm{~m} / \mathrm{s}^{3}\right.$ (beat $\left.\left.\left./ \mathrm{min}\right)\right]\right)$. Figure 5 contains the $\mathrm{WD}_{1}$-HR relation with the greatest slope, that with the smallest slope, and those with moderate slopes.

\section{Discussion}

It has been reported that the basic property of the forcefrequency effect to progressively enhance myocardial contractility as the heart rate increases is markedly augmented due to $\beta$-adrenergic stimulations induced by exercise in normal hearts $[12,13]$. This possibility was examined in conscious dogs through the study of forcefrequency effects during graded infusions of the $\beta$-adrenergic receptor agonist dobutamine under resting conditions. During infusions of low, medium, and high doses of dobutamine, the heart rate varied from 100 to $210 \mathrm{bpm}$ by atrial pacing. During the dobutamine infusions, LV peak $\mathrm{d} P / \mathrm{d} t$ progressively increased as the heart rate was augmented; the increase was greater at high doses than at low doses of dobutamine [14]. Thus, the regulatory effect of enhanced $\beta$-adrenergic stimulation on the FFR is as important as the basal FFR during exercise [15]. Significant impairment of $\beta$-adrenergic amplification of the FFR has
Fig. 5 Relations between the maximum values of $\mathrm{WD}\left(\mathrm{WD}_{1}\right)$ and heart rate (HR) (bpm). As representative data, $\mathrm{WD}_{1} \mathrm{HR}$ relation with the greatest slope, that with the smallest slope, and those with moderate slopes are shown. The solid lines indicate the regression lines. The dotted curves show the $95 \%$ confidence bands of the regression lines
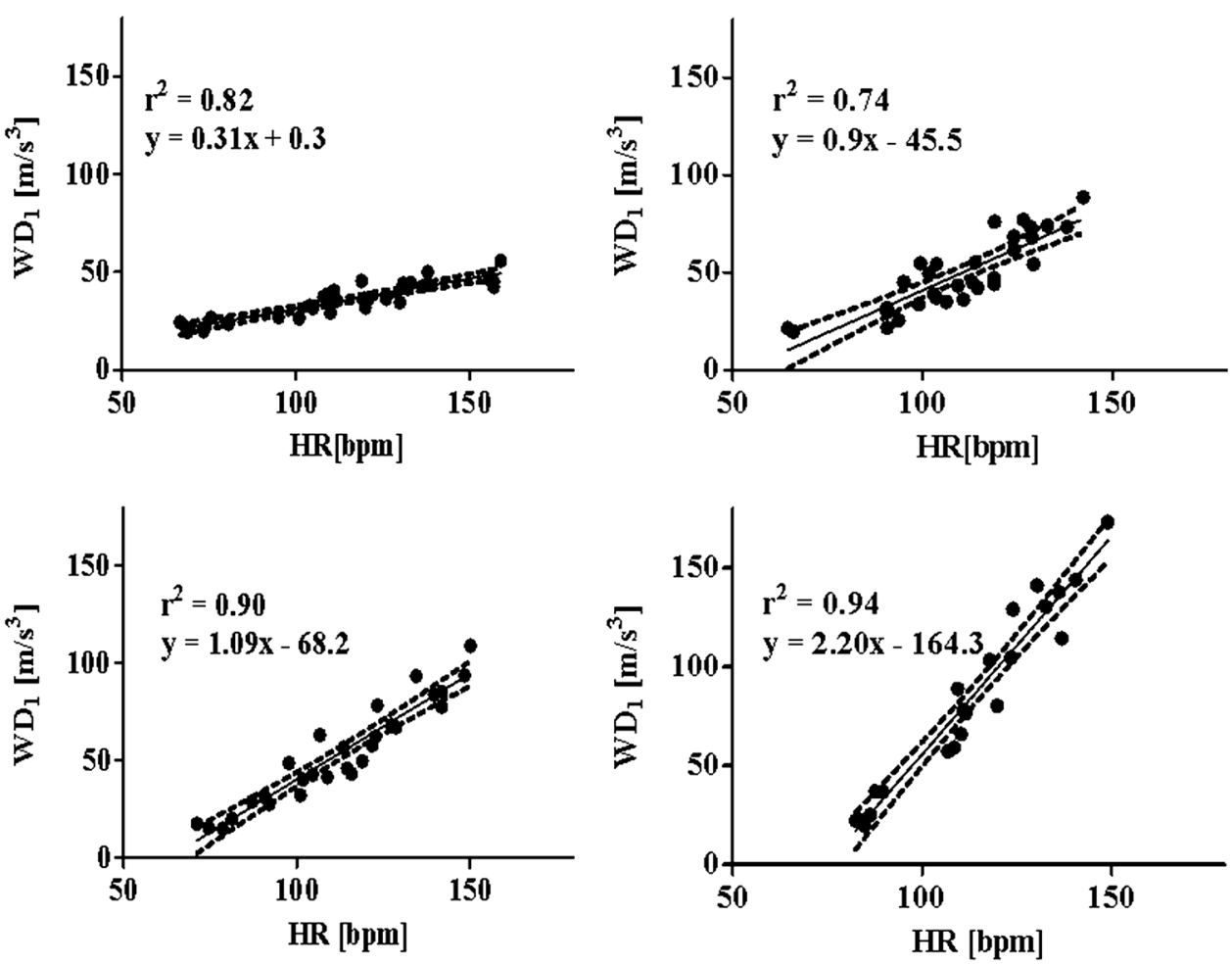
been observed in heart failure, which could contribute to impaired cardiac contractility during exercise [16, 17]. Accordingly, one would expect that the diversity of responses of the FFR to $\beta$-adrenergic stimulations induced by exercise affords increased sensitivity for detection of contractile impairment.

One group has used exercise echocardiography to demonstrate FFRs noninvasively [18]. However, in contrast to the present study, their index (systolic blood pressure/end-systolic volume) was a rarely used and unvalidated index of contractility.

Several other groups have used tissue Doppler-derived isovolumic acceleration (IVA) during exercise, and presented FFR curves [19-21]. However, IVA is a parameter of regional myocardial contractility of the LV wall, and does not necessarily represent the global contractile state of the left ventricle. Indeed, IVA values vary considerably according to the region of interest of the LV wall. In some cases, IVA obtained from the mid lateral wall is more than $50 \%$ greater than that obtained from the basal anterior wall [22]. In addition, the IVA-HR relation during exercise is not linear [21], in contrast to the linear $\mathrm{WD}_{1}-\mathrm{HR}$ relation. It is always easier to characterize a linear relation than to characterize a nonlinear relation.

Equation 4 shows that WD contains $\beta$. It is widely known that $\beta$ increases with age. However, our study subjects only ranged from 19 to 23 years old. Therefore, $\beta$ was considered to vary only slightly with the individual. In spite of this, the slope of the FFR varied relatively widely. This is considered to show that the FFR during exercise is determined mainly by the cardiac contractile state rather than arterial conditions.

\section{Limitations}

Although the $\mathrm{WD}_{1}$-HR relation depends on age, our study subjects only ranged from 19 to 23 years old. We should enroll a greater number of subjects to be divided into age groups in a future study. The final goal of our study is to apply the force-frequency relation to cardiac rehabilitation. However, we did not enroll patients with heart diseases in the present feasibility study.

\section{Conclusions}

Measurements of carotid arterial wave intensity by echocardiography during exercise give the FFR noninvasively. This will be useful for evaluation of the contractile state of the left ventricle during exercise for cardiac rehabilitation.
Conflict of interest We declare that we have no conflict of interest in connection with this paper.

Ethical considerations All procedures followed were in accordance with the ethical standards of the responsible committee on human experimentation (institutional and national) and with the Helsinki Declaration of 1975, as revised in 2008 (5). Informed consent was obtained from all patients for being included in the study.

\section{References}

1. Anderson PA, Manring A, Johnson EA. Force-frequency relationship. A basis for a new index of cardiac contractility? Circ Res. 1973;33:665-71.

2. Anderson PA, Rankin JS, Arentzen CE, et al. Evaluation of the force-frequency relationship as a descriptor of the inotropic state of canine left ventricular myocardium. Circ Res. 1976;39:832-9.

3. Singal PK, Prasad K. Influence of ouabain on the force-frequency relationship in cardiac muscle. Jpn Heart J. 1979;20:341-50.

4. Szigligeti P, Pankucsi C, Banyasz T, et al. Action potential duration and force-frequency relationship in isolated rabbit, guinea pig and rat cardiac muscle. J Comp Physiol B. 1996;166: $150-5$.

5. Schmidt U, Schwinger RH, Bohm M. Halothane restores the altered force-frequency relationship in failing human myocardium. Anesthesiology. 1995;82:1456-62.

6. Monasky MM, Janssen PM. The positive force-frequency relationship is maintained in absence of sarcoplasmic reticulum function in rabbit, but not in rat myocardium. J Comp Physiol B. 2009;179:469-79.

7. Hasenfuss G, Holubarsch C, Hermann HP, et al. Influence of the force-frequency relationship on haemodynamics and left ventricular function in patients with non-failing hearts and in patients with dilated cardiomyopathy. Eur Heart J. 1994;15:164-70.

8. Miura T, Miyazaki S, Guth BD, et al. Influence of the forcefrequency relation on left ventricular function during exercise in conscious dogs. Circulation. 1992;86:563-71.

9. Ohte N, Narita H, Sugawara M, et al. Clinical usefulness of carotid arterial wave intensity in assessing left ventricular systolic and early diastolic performance. Heart Vessels. 2003;18:107-11.

10. Sugawara M, Niki K, Ohte N, et al. Clinical usefulness of wave intensity analysis. Med Biol Eng Comput. 2009;47:197-206.

11. Niki K, Sugawara M, Chang D, et al. A new noninvasive measurement system for wave intensity: evaluation of carotid arterial wave intensity and reproducibility. Heart Vessels. 2002;17: $12-21$.

12. Mattera GG, Vanoli E, Martinez V, et al. Adrenergic effects on force-frequency relationship: a pivotal role for the cardiac intrinsic systems. Acta Physiol (Oxf). 2011;202:141-9.

13. Izawa $\mathrm{H}$, Yokota $M$, Takeichi $Y$, et al. Adrenergic control of the force-frequency and relaxation-frequency relations in patients with hypertrophic cardiomyopathy. Circulation. 1997;96: 2959-68.

14. Kambayashi M, Miura T, Oh B-H, et al. Enhancement of the force-frequency effect on myocardial contractility by adrenergic stimulation in conscious dogs. Circulation. 1992;86:572-80.

15. Ross JJr, Miura T, Kambayashi M, et al. Adrenergic control of the force-frequency relation. Circulation. 1995;92:2327-32.

16. Ross J Jr. Adrenergic regulation of the force-frequency effect. Basic Res Cardiol. 1998;93:95-101 (Epub 1998/12/02).

17. Matsuzaki M, Patritti J, Tajimi T, et al. Effects of beta-blockade on regional myocardial flow and function during exercise. Am J Physiol. 1984;247:H52-60. 
18. Agricola E, Bombardini T, Oppizzi M, et al. Usefulness of latent left ventricular dysfunction assessed by Bowditch Treppe to predict stress-induced pulmonary hypertension in minimally symptomatic severe mitral regurgitation secondary to mitral valve prolapse. Am J Cardiol. 2005;95:414-7.

19. Roche SL, Vogel M, Pitkanen O, et al. Isovolumic acceleration at rest and during exercise in children normal values for the left ventricle and first noninvasive demonstration of exercise-induced force-frequency relationships. J Am Coll Cardiol. 2011;57: 1100-7.

20. Cheung MM, Smallhorn JF, Vogel M, et al. Disruption of the ventricular myocardial force-frequency relationship after cardiac surgery in children: noninvasive assessment by means of tissue Doppler imaging. J Thorac Cardiovasc Surg. 2006;131:625-31.

21. Vogel M, Cheung MM, Li J, et al. Noninvasive assessment of left ventricular force-frequency relationships using tissue Dopplerderived isovolumic acceleration: validation in an animal model. Circulation. 2003;107:1647-52.

22. Pauliks LB, Vogel M, Madler CF, et al. Regional response of myocardial acceleration during isovolumic contraction during dobutamine stress echocardiography: a color tissue Doppler study and comparison with angiocardiographic findings. Echocardiography. 2005;22:797-808. 